Revista Brasileira de Agricultura Irrigada v.12, nº.4, p. 2749 - 2754, 2018

ISSN 1982-7679 (On-line)

Fortaleza, CE, INOVAGRI - http://www.inovagri.org.br

DOI: $10.7127 /$ rbai.v12n400970

Protocolo 970.18 - 15/06/2018 Aprovado em 21/06/2018

\title{
DESENVOLVIMENTO DE UM ALGORITMO COMPUTACIONAL PARA DETERMINAÇÃO DO ESPAÇAMENTO ENTRE DRENOS SUBTERRÂNEOS PELO MÉTODO DE HOOGHOUT
}

\author{
Eugenio Paceli de Miranda ${ }^{1}$, Gilbenes Bezerra Rosal ${ }^{2}$, Rayane De Morais Furtado ${ }^{3}$, Tatiana \\ Belo de Sousa Custódio ${ }^{4}$
}

\section{RESUMO}

Foi desenvolvido um algoritmo computacional em linguagem Visual Basic for Application (VBA) para a determinação do espaçamento entre drenos subterrâneos. Utilizou-se a metodologia de Hooghout para regimes permanentes, para solos homogêneos, que apresentam uma única condutividade hidráulica e para solos estratificados, que apresentam duas camadas com condutividades hidráulicas diferentes. Os resultados obtidos com o algoritmo foram comparados com os fornecidos pela literatura. A diferença média entre os resultados fornecidos pelo algoritmo e a literatura foi de $2,76 \%$, sendo que alguns resultados obtidos usando o algoritmo foram exatamente iguais àqueles.

Palavras-chave: Drenagem agrícola, lençol freático, condutividade hidráulica.

\section{DEVELOPMENT OF A COMPUTATIONAL ALGORITHM TO DETERMINATION OF UNDERGROUND DRAIN SPACE BY HOOGHOUT METHOD}

\begin{abstract}
A computational algorithm was developed in Visual Basic for Application (VBA) language to determine the spacing between underground drains. The Hooghout methodology was used for permanent regimes for homogeneous soils, with a single hydraulic conductivity and for stratified, soils with two layers with different hydraulic conductivities. The results obtained with the algorithm were compared with those provided by the literature. The mean difference between the results was $2.76 \%$, and some results obtained using the algorithm were the same as those presented in the literature.
\end{abstract}

Keywords: Agricultural drainage, groundwater, hydraulic conductivity.

\footnotetext{
${ }^{1}$ Prof. Doutor IFCE, Iguatu - Ceará, Email: eu.paceli@yahoo.com.br

${ }^{2}$ Graduando em Tecnologia em Irrigação e Drenagem, IFCE, campus Iguatu. Email: gilbenesbezerrarosal@gmail.com

${ }^{3}$ Graduando do curso Tecnologia em Irrigação e Drenagem, IFCE, campus Iguatu-Ceará.

${ }^{4}$ Graduando do curso Tecnologia em Irrigação e Drenagem, IFCE, campus Iguatu-Ceará.
} 


\section{INTRODUÇÃO}

A drenagem consiste na retirada do excesso de água do solo a uma taxa que permita uma exploração econômica das culturas e a utilização da área, por longo tempo (Pompeu et al., 2016). Isso é feito através da instalação de drenos que recebem a água do perfil do solo, onde são instalados. É uma prática trabalhosa e muito cara, que requer um processo matemático relativamente complexo para o dimensionamento de drenos.

Para evitar gastos futuros com drenagem do solo é necessário que se planeje o manejo antes mesmo de observar os possíveis problemas, para isso têm-se a necessidade de recorrer a conhecimentos técnicos e aplicar em campo.

Em terras agrícolas, com déficit de drenagem, torna-se importante a remoção do excesso de água por estruturas artificiais, denominadas drenos, melhorando as condições de aeração, estrutura e resistência do solo. Porém, para obter um adequado funcionamento do sistema é importante considerar os diversos fatores envolvidos, como: características do solo, da cultura, do regime de escoamento e os critérios de drenagem. (Ludwig, 2013).

Na drenagem agrícola, os drenos são instalados, geralmente, sobre a camada impermeável ou a certa distância acima desta. A profundidade de instalação dos drenos e a do perfil, a condutividade hidráulica e a porosidade drenável são parâmetros de importância no cálculo do espaçamento de drenos. Diversas equações são sugeridas para o cálculo deste espaçamento, porém não há uma única equação que se adapte bem a todas as situações. DUARTE et al., (2001).

No dimensionamento, podem-se utilizar as equações de regime permanente, as quais são empregadas quando objetiva-se manter o nível do lençol freático constante no tempo e no espaço, sendo indicado apenas para regiões com chuvas de baixa intensidade e longa duração (Ludwig, 2013).

Millar (1988) considera que no dimensionamento de um sistema de drenagem um dos fatores mais importantes é a distância entre os drenos. $\mathrm{O}$ dimensionamento hidráulico nos sistemas de drenagem agrícola deve-se conhecer a precipitação da região, suportando valor extremo de precipitação que apresenta segurança para os valores usuais. MESQUITA et al., (2009); SANTOS et al., (2014); GUIMARÃES et al., (2015).

O objetivo do presente trabalho foi estimar 0 espaçamento entre drenos subterrâneo por meio de um algoritmo computacional utilizando o método de Hooghout.

\section{MATERIAL E MÉTODOS}

Foi desenvolvido um algoritmo computacional em linguagem Visual Basic for Application (VBA) para determinação do espaçamento entre drenos subterrâneos pelo método de Hooghout.

O algoritmo fornece o espaçamento entre drenos subterrâneos para duas condições de perfil do solo, para perfil homogêneo e para perfil estratificado. A Equação 1 é utilizada para o caso do perfil do solo homogêneo, que apresenta a mesma condutividade hidráulica. E a Equação 2 para um perfil estratificado em dois horizontes, com duas condutividades hidráulicas.

$$
\begin{aligned}
L^{I} & =\frac{4 K h^{2}+8 K d h}{R} \\
L^{I I} & =\frac{4 K_{1} h^{2}+8 K_{2} d h}{R}
\end{aligned}
$$

em que: $L^{\mathrm{I}}$ e $\mathrm{L}^{\mathrm{II}}$ - espaçamento entre drenos (m) para 1 e 2 condutividades hidráulicas, respectivamente; $\mathrm{K}$ - condutividade hidráulica do solo ( $\left.\mathrm{m} \mathrm{dia}^{-1}\right) ; \mathrm{h}$ - altura da água na metade dos drenos (m); d - estrato equivalente (m) e $\mathrm{R}$ - recarga (m dia $\left.{ }^{-1}\right)$.

$O$ estrato equivalente (d) é determinado pela Equação 3 também proposta por Hooghougt.

$$
d=\frac{L}{2,55 \cdot\left(\frac{D}{L}\right) \cdot \operatorname{Ln} \cdot\left(\frac{D}{P M}\right)+1}
$$


em que: $\mathrm{d}$ - estrato equivalente (m); $\mathrm{L}-$ espaçamento entre drenos (m); D - distância do dreno à camada impermeável (m) e PM perímetro molhado do dreno $(\mathrm{m})$.
Para verificar o desempenho do algoritmo comparou-se com resultados apresentados pela literatura, conforme Tabela 1 .

Tabela 1. Parâmetros hidráulicos para a determinação do espaçamento entre drenos subterrâneos fornecidos pela literatura.

\begin{tabular}{lcccccc}
\hline \multicolumn{1}{c}{ Autores } & $\begin{array}{c}\mathrm{h} \\
(\mathrm{m})\end{array}$ & $\begin{array}{c}\mathrm{D} \\
(\mathrm{m})\end{array}$ & $\begin{array}{c}\mathrm{r} \\
(\mathrm{m})\end{array}$ & $\begin{array}{c}\mathrm{K}_{1} \\
\left(\mathrm{~m} \mathrm{dia}^{-1}\right)\end{array}$ & $\begin{array}{c}\mathrm{K}_{2} \\
\left(\mathrm{~m} \mathrm{dia}^{-1}\right)\end{array}$ & $\begin{array}{c}\mathrm{R} \\
\left(\mathrm{mm} \mathrm{dia}^{-1}\right)\end{array}$ \\
\hline Duarte et al. (2015) & 0,7 & 1,9 & 0,05 & 1,0 & - & 6,0 \\
Costa (2008) & 0,4 & 2,0 & 0,05 & 0,3 & 1,2 & 12,0 \\
Mello (2009) & 0,4 & 2,0 & 0,05 & 0,25 & 1,5 & 15,0 \\
Melo Souza (2009) & 0,5 & 3,9 & 0,10 & 1,0 & - & 7 \\
Millar (1988) & 0,6 & 3,0 & 0,10 & 0,5 & 1,0 & 5 \\
Bernardo (2009) & 0,5 & 3,4 & 0,075 & 0,86 & - & 5 \\
\hline
\end{tabular}

\section{RESULTADOS E DISCUSSÃO}

Na Figura 1 é mostrada a janela inicial do algoritmo. Nela estão os botões de opções para que o usuário escolha entre o caso de solo homogêneo ou estratificado.

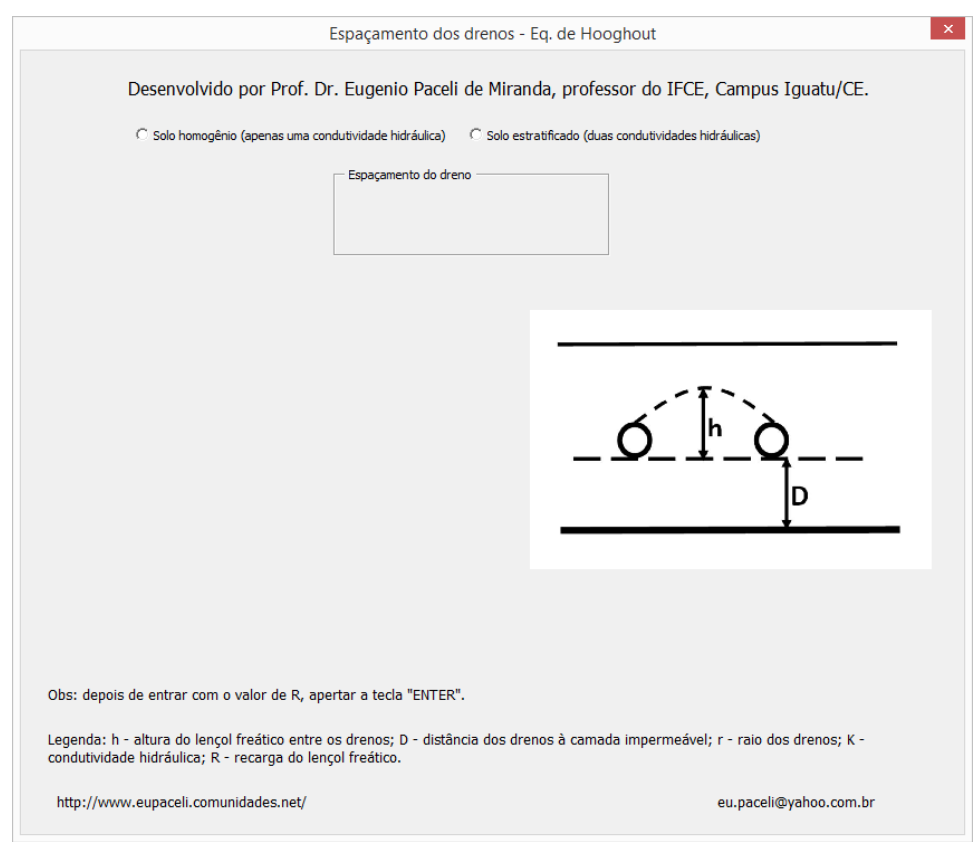

Figura 1. Janela inicial do algoritmo.

As Figuras 2 e 3 mostram, respectivamente, as interfaces do programa para o caso de camada homogênea, quando o perfil do solo apresenta apenas uma condutividade hidráulica (Figura 1) e para o caso de solo estratificado, quando o perfil do solo apresenta duas condutividades hidráulicas (Figura 2). 


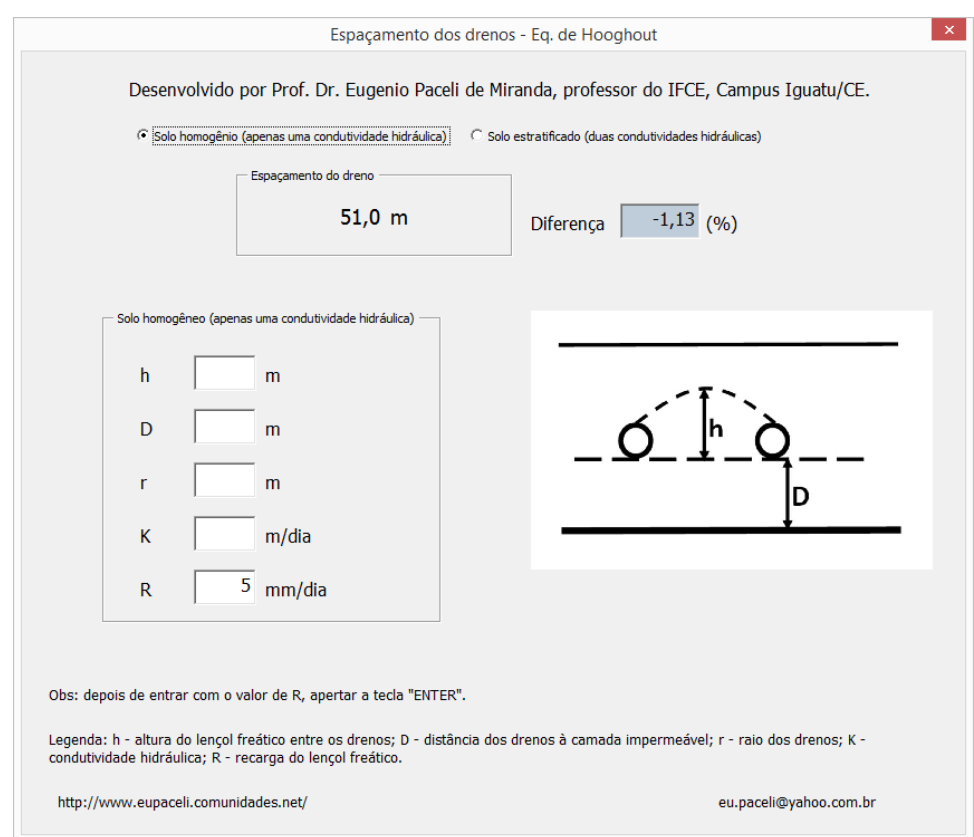

Figura 2. Interface do programa para o caso de solo horizonte homogêneo.

Escolhido o caso específico, são mostrados os campos para os parâmetros de entrada do algoritmo: h (m) - altura do lençol freático entre os drenos; D (m) - distância dos drenos à camada impermeável; $\mathrm{r}(\mathrm{m})$ - raios dos drenos; K (m/dia) - condutividade hidráulica do solo, para o caso de solo homogêneo, com uma só condutividade hidráulica, e K1 e K2, para as condutividades da primeita e da segunda camada, no caso de solo de perfil estratificado, e R (mm/dia) recarga do lençol freático.

Informado o valor do último parâmetro, o algoritmo mostra o resultado do espaçamento entre drenos na parte superior da janela.

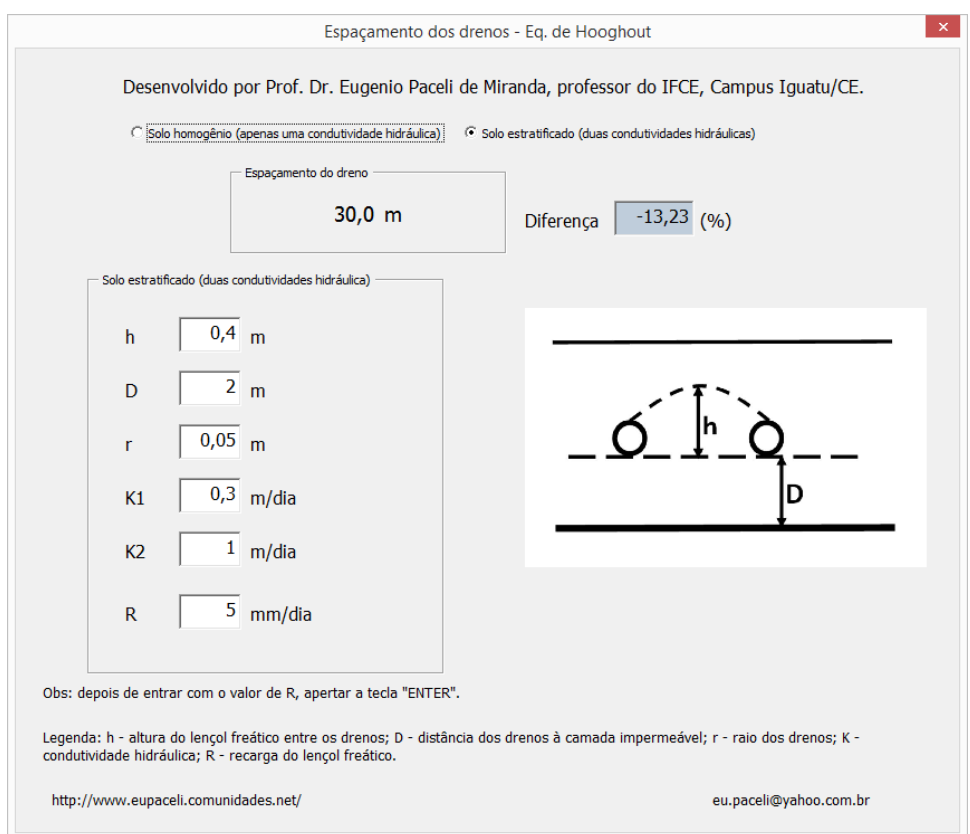

Figura 3 . Interface do programa para o caso de solo horizonte estratificado em duas camadas.

Na Tabela 2 são mostrados os resultados das simulações feitas usando o algoritmo, os apresentados pela literatura e a diferença entre eles. 


\section{DESENVOLVIMENTO DE UM ALGORITMO COMPUTACIONAL PARA DETERMINAÇÃO DO ESPAÇAMENTO ENTRE DRENOS SUBTERRÂNEOS PELO MÉTODO DE HOOGHOUT}

Como pode ser observado na Tabela 2 os resultados obtidos com o algoritmo, a maior diferença foi 9,8\%, em relação ao apresentado por Duarte et al., (2015), a menor diferença foi de zero, ou seja, os resultados foram iguais aos apresentados por Costa (2008) e Mello (2009). A diferença média entre o algoritmo e os apresentados pela literatura foi de $2,76 \%$.

Tabela 2. Resultados apresentados pela literatura e pelo Algoritmo.

\begin{tabular}{lccc}
\hline \multicolumn{1}{c}{ Literatura/Autores } & Literatura & Algoritmo & Variação (\%) \\
\hline Duarte et al. (2015) & 37,0 & 41,0 & 9,8 \\
Costa (2008) & 20,0 & 20,0 & 0 \\
Mello (2009) & 20,0 & 20,0 & 0 \\
Melo Souza (2009) & 36,2 & 39,0 & 7,2 \\
Millar (1988) & 48,0 & 47,0 & 4,7 \\
Bernardo (2009) & 52,0 & 51,0 & 1,9 \\
\hline
\end{tabular}

\section{CONCLUSÃO}

O algoritmo desenvolvido obteve resultados iguais ou muito próximos aos apresentados pela literatura, mostrando que o algoritmo computacional pode ser usado para a determinação do espaçamento entre drenos subterrâneos.

\section{REFERÊNCIAS}

BERNARDO, S.; SOARES, A.A.; MANTOVANI, E.C. Manual de Irrigação. $8^{a}$ Edição. Editora UFV. 2009.

COSTA, R.N.T. Drenagem Agrícola. Apostila. Universidade Federal do Ceará, Departamento de Engenharia Agrícola. Fortaleza, Ceará. 2008.

DUARTE, S.N.; SILVA, $\quad$ E.F.F.; MIRANDA, J.H.; MEDEIROS, J.F.; COSTA, R.N.T.; GHEYI, H.R. Fundamentos de Drenagem Agrícola. INCTSal. Fortaleza, Ceará, 2015.

DUARTE S.N.; CRUCIANI D.E.; CARARO D.C.; PASSOS K.S.; LOPES A D.V. Avaliação de equações de drenagem para regime não permanente em condições de laboratório. Revista Brasileira de Engenharia Agrícola e Ambiental, v.5, n.2, p.181-186, Campina Grande, PB, 2001.

GUIMARÃES, M. J. M.; LOPES, I. ; SILVA, W. P. ; SILVA, A. S. . Estudo das precipitações máximas para o município de Cruz das Almas, BA. Revista Magistra, v. 27, p. 326-332, 2015.

LUDWIG R.; SAAD J.C.C.; PUTTI F.F.; JUNIOR J.F. DA S.; SCHIMIDT P.R.A.; LATORRE D. DE O.; SILVA I. P. DE F. E. Dimensionamento de sistemas de drenagem através de software computacional. Brazilian Journal of Biosystems Engineering, 7 (2), 70-76, 2013.

MELLO, J.L.P. Drenagem Agrícola. Apostila. Universidade Federal do Rio de Janeiro. Departamento de Engenharia. Rio de Janeiro. 2009.

MELO SOUZA, R. O. R. Disciplina: Irrigação e Drenagem. Apostila. Universidade Federal Rural da Amazônia. Instituto de Ciências Agrárias. Pará, 2009.

MESQUITA W. O., GRIEBELER, N P., \& OLIVEIRA, L. F. C. Precipitações máximas diárias esperadas para as regiões central e sudeste de Goiás. Pesquisa Agropecuária Tropical, 39 (2), 73-81, 2009. 
MILLA, A.A. Drenagem de Terras Agrícolas: bases agronômicas. Editerra Editorial. São Paulo. 1988.

POMPEU, FSS ; MIRANDA, E. P. ; OLIVEIRA, FER ; MARTINS, G. S. ; MACIEL, W. M. Ensaio comparativo de envoltórios de drenos tubulares na drenagem agrícola. Magistra, v. 28, p. 522-529, 2016

SANTOS, W. O., MESQUITA, F. O., BATISTA, B. D. O., BATISTA, R. O., \& ALVES, A.S. Precipitações máximas para o município de Mossoró de 1964 a 2011 pela distribuição de Gumbel. Irriga, 9 (2), 207-213, 2014. 